\title{
Lateral composition modulation in mixed anion multilayers
}

\author{
C. Dorin and J. Mirecki Millunchick ${ }^{\text {a) }}$ \\ Department of Materials Science and Engineering, University of Michigan, Ann Arbor, \\ Michigan 48109-2136 \\ C. Wauchope \\ Electron Microbeam Analysis Laboratory, University of Michigan, Ann Arbor, Michigan 48109-2143
}

(Received 7 May 2002; accepted 4 September 2002)

\begin{abstract}
Lateral composition modulation on the group V sublattice has been observed in $\mathrm{GaAs} / \mathrm{GaSb}$ short period superlattices. Cross sectional transmission electron microscopy and x-ray diffraction reciprocal space maps reveal that all structures are phase-separated with $\mathrm{Sb}$ compositions for the strongest modulated structure of $x=0.73$ in the Sb-rich regions, $x=0.55$ in the As-rich regions, and wavelengths $15 \leqslant \Lambda \leqslant 20 \mathrm{~nm}$. The composition modulation observed in these films is not due to spinodal decomposition, because an alloy grown at the same conditions results in a homogeneous layer, but may be related to vertical stacking of quantum dots that nucleate during the growth of the structure. (C) 2002 American Institute of Physics. [DOI: 10.1063/1.1517712]
\end{abstract}

Compositional nonuniformities and phase separation in alloy layers of compound semiconductor films have large ramifications in the resultant device properties; ${ }^{1}$ therefore, it is critical to understand and control these phenomena. Phase separation can spontaneously form parallel or perpendicular to the growth direction. Lateral composition modulation, in which the phase separation is perpendicular to the growth direction, has been reported in alloys such as InAlAs/InP, 2,3 GaAsN/GaAs, ${ }^{4}$ and InGaP/GaAs. ${ }^{5}$ Vertical composition modulation has been reported in alloys such as $\mathrm{InGaP} / \mathrm{GaAs}^{6}$ and InAsSb/GaAs. ${ }^{7}$ For example, it was found that $\operatorname{InAs}_{0.5} \mathrm{Sb}_{0.5}$ grown at $430{ }^{\circ} \mathrm{C}$ produced a homogeneous random alloy, while the same material grown at $340^{\circ} \mathrm{C}$ was strongly modulated along the (001) growth direction. This phenomenon has been attributed to the presence of a miscibility gap at these low growth temperatures. ${ }^{7}$

Lateral composition modulations are observed to be regular and robust for short period superlattices (SPSs) in which each layer is on the order of a monolayer (ML) or two, and the layers have alternating compressive and tensile strain with respect to the substrate. A great deal of attention has been paid to mixed cation systems such as GaAs/InAs, ${ }^{8}$ AlAs/InAs, ${ }^{9}$ and GaP/InP SPSs,${ }^{10}$ in which the composition modulation occurs on the group III sublattice. Typical composition modulation wavelengths are on the order of $100 \AA$ $<\Lambda<400 \AA,{ }^{11}$ and the modulation amplitude varies by $20 \%$. ${ }^{12}$ To date, however, lateral composition modulation has not been studied in SPS structures containing both arsenide and antimonide compounds, which may be beneficial for thermophotovoltaic applications. ${ }^{1}$ In this letter, we report the formation of lateral composition modulation on the group $\mathrm{V}$ sublattice in SPS structures consisting of $\mathrm{GaAs} / \mathrm{GaSb}$ multilayers.

All growths were carried out in a molecular beam epitaxy (MBE) chamber equipped with solid sources for Ga and In and valved cracking cells for $\mathrm{Sb}$ and As. The samples were grown upon InP (001) substrates that were prepared by

${ }^{a)}$ Electronic mail: joannamm@umich.edu heating at $530{ }^{\circ} \mathrm{C}$ under $\mathrm{As}_{4}$ overpressure. A nominally latticed-matched InGaAs buffer layer was deposited at a temperature $T=500{ }^{\circ} \mathrm{C}$. The SPSs were deposited at $T$ $=400^{\circ} \mathrm{C}$ using $\mathrm{Sb}_{2}$ and $\mathrm{As}_{4}$ species. The GaAs and $\mathrm{GaSb}$ growth rates were calibrated by reflection high energy electron diffraction (RHEED) oscillations prior to growth and were 0.18 and $0.21 \mathrm{ML} / \mathrm{s}$, respectively. The ratio of $\mathrm{Sb}_{2}$ to $\mathrm{As}_{4}$ beam equivalent pressures (BEP) was approximately 0.3 , and the total group V BEP was $1 \times 10^{-5}$ Torr for all the samples in this study, except where noted. Several different SPS structures and $\mathrm{GaAs}_{1-x} \mathrm{Sb}_{x}$ alloy layers were examined.

The samples were examined by a battery of in situ and ex situ characterization techniques. The quality of the crystalline surface was monitored in situ by RHEED. Double crystal X-ray diffraction (XRD) $\theta / 2 \theta$ scans and reciprocal space maps were recorded with $\mathrm{Cu} K_{\alpha}$ radiation in order to characterize the microstructure of the SPS. Cross section transmission electron microscopy (XTEM) was also performed in order to corroborate the XRD data. Samples were prepared using mechanical thinning, followed by Ar ion milling at $4.5 \mathrm{kV}$ and angles between $3^{\circ}$ and $5^{\circ}$. The buildup of In metal islands on the surface was confined to the substrate and did not affect the sample quality in the region of interest. XTEM studies were carried out on a field emission TEM (JEOL 2010FX) operated at $200 \mathrm{kV}$. High angle annular dark field (HAADF) scanning transmission electron microscopy (STEM) imaging was used to collect images with high mass-thickness contrast and little to no diffraction contrast, allowing direct imaging of the composition modulation. $\mathrm{X}$-ray energy dispersive spectroscopy (XEDS) line profiles were collected using a software controlled STEM probe with drift correction.

In all cases, the growth of the GaAs/GaSb SPS structures resulted in three-dimensional (3D) islanding and lateral composition modulation to some degree. For example, the RHEED pattern for a $\mathrm{GaAs}_{2 \mathrm{ML}} / \mathrm{GaSb}_{2 \mathrm{ML}}$ structure, where each layer is 2 ML thick, degraded into spots during the growth of either layer, indicating 3D islanding. The final RHEED pattern was dim and spotty signifying a rough and 

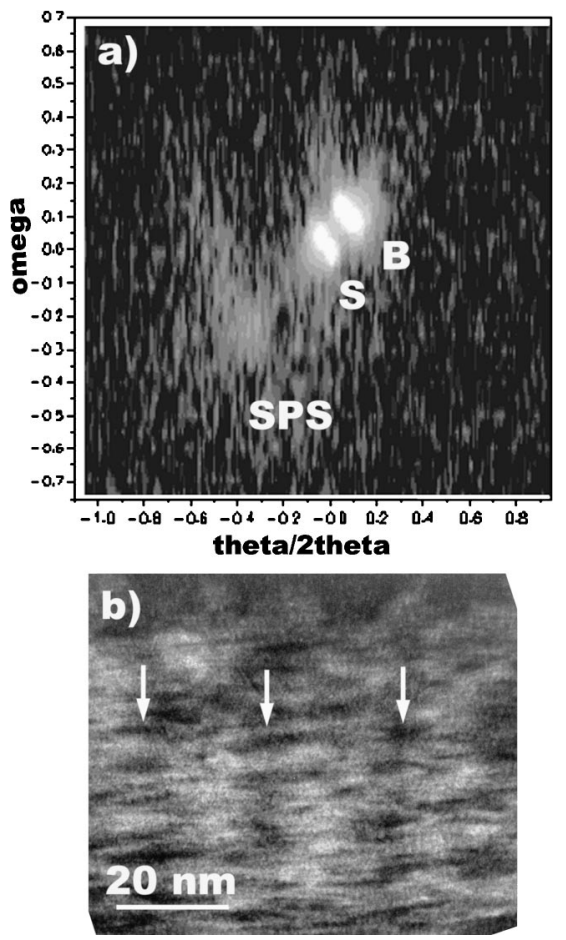

FIG. 1. (a) (224) XRD reciprocal space map showing the substrate (S), buffer layer (B), and SPS peak for a $\mathrm{GaAs}_{2 \mathrm{ML}} / \mathrm{GaSb}_{2 \mathrm{ML}}$ SPS. (b) (002) DF XTEM image of the same sample. The arrows denote compositionally modulated regions that result due to vertical stacking of quantum dots.

disordered final surface. The average $\mathrm{Sb}$ composition of the SPS was found to be $x=0.55$, as determined from (004) XRD rocking curves. The (224) XRD reciprocal space map [Fig. 1(a)] shows diffuse lateral satellite peaks, which suggests the presence of lateral composition modulation along the [110] direction. ${ }^{13}$ Figure 1(b) shows an (002) XTEM dark field micrograph of this sample. Discontinuous platelets are visible in this image (denoted by arrows) that may be interpreted as vertically stacked quantum dots. This is consistent with the spotty RHEED pattern observed during growth and may explain the appearance of lateral satellites observed by $\mathrm{XRD}$. Indeed, image contrast is visible in HAADF images due to composition variations (not shown).

In order to improve the microstructure of the laterally modulated films, another $\mathrm{GaSb}_{2 \mathrm{ML}} / \mathrm{GaAs}_{2 \mathrm{ML}}$ structure was grown with an additional $4 \mathrm{~s}$ Sb soak prior to the deposition of GaSb. That is, the GaAs surface was exposed to an overpressure of $\mathrm{Sb}_{2}$ for $4 \mathrm{~s}$ prior to deposition of $\mathrm{GaSb}$. The RHEED showed chevrons during the deposition of the GaAs layer, and recovered to a streaky $3 \times 1$ surface reconstruction during the deposition of the GaSb layers, as previously. The XRD reciprocal space map again shows evidence of weak lateral satellites (not shown), and the average Sb composition is $x=0.58$, indicating that the Sb soak step resulted in additional Sb incorporation. Figure 2(a) is a (002) dark field micrograph of this sample. Platelets are discernable in higher magnification images (not shown), in addition to irregular contrast variations and occasional defects. The platelets form due to islanding during the deposition of GaAs layer, but may also be attributed to the microstructure of the wetting layer. It has been shown that the first monolayer of GaSb on GaAs consists of plate-like islands with dimensions of approximately $10 \mathrm{~nm},{ }^{14}$ which is of the same order of magnitude as the composition modulation observed in these films.

The contrast in this micrograph is due to both variations in composition and strain, therefore, HAADF images were acquired to discern the image contrast due to differences in composition. Figure 2(b) presents an HAADF image of this sample. Regular, periodic lateral composition modulation is clearly visible in the image. The dark bands correspond to As-rich regions and the light bands correspond to Sb-rich regions. The inset shows a higher magnification image of an As-rich column. It is clear from this image that the column arises due to vertical stacking of GaAs dots in a GaSb matrix. This is consistent with RHEED observations during growth. XEDS [Fig. 2(c)] shows that the As and Sb compositions vary sinusoidally with a periodicity $\Lambda=15 \mathrm{~nm}$ and are out of phase with one another, confirming compositional modulation on the group V sublattice. The $\mathrm{Sb}$ composition was determined from the XEDS line profile to be $x=0.73$ in the Sb-rich region, and $x=0.55$ in the As-rich region. It is surprising, however, that the Ga composition also oscillates in phase with As. Since all the samples were grown with a InGaAs buffer layer, one possible explanation is that In segregates into the layer and phase separates from the $\mathrm{Ga}$ on the group III sublattice. However, no traces of In were found in the SPSs. Furthermore, In segregation is negligible at this growth temperature. Another possible explanation is that the $\mathrm{Ga}$ oscillation is due to excess $\mathrm{Sb}$ atoms in interstitial sites or occupying Ga sites. ${ }^{15}$

The presence of lateral composition modulation in these films is not due to spinodal decomposition, despite the presence of a miscibility gap in GaAsSb alloys at this growth temperature. ${ }^{16}$ To demonstrate this, a $\mathrm{GaAs}_{1-x} \mathrm{Sb}_{x}$ random alloy was grown under the same growth conditions as sample
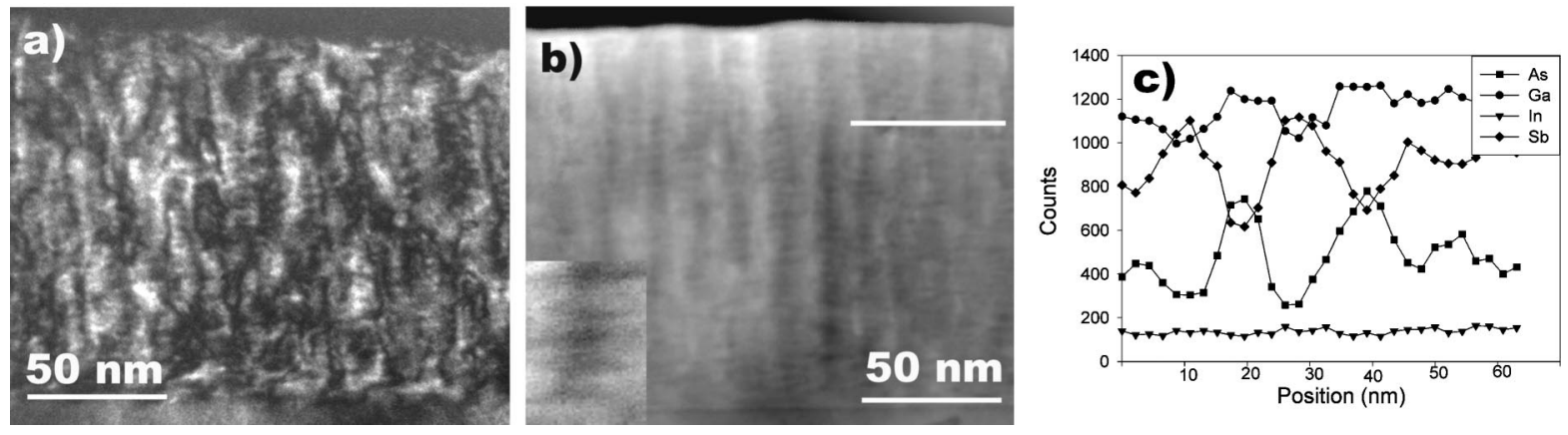
F193, that is, rather than depositing a multilayer structure, all shutters were opened at once. The XRD (224) reciprocal space map for this film shows no lateral satellites, suggesting that the alloy is structurally homogeneous. However, the film peak is broadened along $\omega$, indicative of a film with a large density of dislocations, ${ }^{17}$ which was confirmed by XTEM. (224) glancing incidence and glancing exit XRD measurements show that the alloy is fully relaxed and that $x=1$; that is, no As incorporation has occurred. This composition was confirmed by XEDS. Another $\mathrm{GaAs}_{1-x} \mathrm{Sb}_{x}$ random alloy was also grown at same temperature and $\mathrm{Sb} / \mathrm{As} \mathrm{BEP}$ ratio but lower total group $\mathrm{V}$ overpressure $\left(2 \times 10^{-6}\right.$ Torr $)$ in order to take into account the lower instantaneous group $\mathrm{V}$ fluxes that are present during the deposition of the multilayer structure. No As incorporation occurred in this film either. It is known that As incorporation in mixed anion ternaries such as $\mathrm{GaAsSb}$ is strongly dependent on the total group V overpressure, growth temperature and group V BEP ratio, and that As incorporation is reduced in the presence of $\mathrm{Sb}$ at high group $\mathrm{V}$ overpressure. ${ }^{18}$ As incorporation in these films may be enhanced by using $\mathrm{As}_{2}$ species, since the cracking of $\mathrm{As}_{4}$ is inefficient at low growth temperatures $\left(T=400^{\circ} \mathrm{C}\right) .{ }^{19}$ To test this, a final alloy was grown under the same conditions but using $\mathrm{As}_{2}$ species. Indeed, XRD shows that the resultant alloy was a high quality homogeneous film with a composition $x=0.5$ and no lateral composition modulation.

The fact that there is significant As incorporation in the SPS structures compared to the random alloy is not unexpected. Because the structure is deposited by alternating layers of binary compounds, the $\mathrm{Sb}$ flux does not hinder As incorporation compared to the deposition of a random alloy. Despite this, As sticking coefficient is clearly less than unity. Therefore, such a multilayer structure may be employed to obtain As incorporation in these alloys when the As sticking coefficient is low.

The presence of lateral composition modulation in the SPS multilayer structures is associated with roughening of the growth front. Previous studies for lateral composition modulation in mixed cation material systems indicate that some degree of surface roughening is correlated to the appearance of lateral composition modulation, however, too much roughening or 3D island formation destroys the regularity of lateral composition modulation. ${ }^{20}$ Experiments $^{21}$ and theory $^{22}$ have shown that the strongest lateral composition modulation occurs for structures grown under an overall tensile strain. Therefore, improved arrays of phase-separated material are expected for GaAs/GaSb SPS structures with higher As content.

Lateral composition modulation has been demonstrated in antimonide-arsenide materials systems. It occurs as a result of the SPS multilayer structure itself and is not due to spinodal decomposition, since a random alloy grown under similar conditions is homogeneous in composition. The lat- eral composition modulation is periodic with typical wavelengths $15 \leqslant \Lambda \leqslant 20 \mathrm{~nm}$ and $\mathrm{Sb}$ compositions of $x=0.73$ for Sb-rich regions and $x=0.55$ for the As-rich regions for the strongest modulated structure. Surprisingly, the Ga composition varies laterally as well, suggesting that $\mathrm{Sb}$ interstitials or antisite defects may be present. The microstructure of the SPS shows that the lateral composition modulation may arise due to vertical stacking of GaAs quantum dots in a matrix of $\mathrm{GaSb}$.

The authors wish to acknowledge useful discussions with Dr. J. Mansfield, Dr. B. Bennett, and K. Matney. This work was supported by NSF Grant DMR-9979952.

${ }^{1}$ C. A. Wang, H. K. Choi, D. C. Oakley, and C. W. Charache, J. Cryst. Growth 195, 346 (1998).

${ }^{2}$ S. W. Jun, T.-Y. Seong, J. H. Lee, and B. Lee, Appl. Phys. Lett. 68, 3443 (1996).

${ }^{3}$ S. Francoeur, M. C. Hanna, A. G. Norman, and A. Mascarenhas, Appl. Phys. Lett. 80, 243 (2002).

${ }^{4}$ I. Suemune, N. Morooka, K. Uesugi, Y.-W. Ok, and T.-Y. Seong, J. Cryst. Growth 221, 546 (2000).

${ }^{5}$ M. Sugisaki, H.-W. Ren, K. Nishi, S. Sugou, and Y. Masumoto, Phys. Rev. B 61, 16040 (2000); D. M. Follstaedt, R. P. Schneider, and E. D. Jones, J. Appl. Phys. 77, 3077 (1994).

${ }^{6}$ D. M. Follstaedt, R. P. Schneider, and E. D. Jones, J. Appl. Phys. 77, 3077 (1994)

${ }^{7}$ T.-Y. Seong, A. G. Norman, I. T. Ferguson, and G. R. Booker, J. Appl. Phys. 73, 8227 (1993)

${ }^{8}$ K. Y. Cheng, K. C. Hsieh, and J. N. Baillargeon, Appl. Phys. Lett. 60, $2892(1992)$

${ }^{9}$ A. G. Norman, S. P. Ahrenkiel, H. Moutinho, M. M. Al-Jassim, A. Mascarenhas, J. Mirecki Millunchick, S. R. Lee, R. D. Twesten, D. M. Follstaedt, J. L. Reno, and E. D. Jones, Appl. Phys. Lett. 73, 1844 (1998).

${ }^{10}$ K. C. Hsieh, J. N. Baillargeon, and K. Y. Cheng, Appl. Phys. Lett. 57, 2244 (1990)

${ }^{11}$ J. Mirecki Millunchick, R. D. Twesten, S. R. Lee, D. M. Follstaedt, E. D. Jones, S. P. Ahrenkiel, Y. Zang, H. M. Cheong, and A. Mascarenhas, MRS Bull. 22, 38 (1997).

${ }^{12}$ R. D. Twesten, D. M. Follstaedt, S. R. Lee, E. D. Jones, J. L. Reno, J. Mirecki Millunchick, A. G. Norman, S. P. Ahrenkiel, and A. Mascarenhas, Phys. Rev. B 60, 13619 (1999).

${ }^{13}$ S. R. Lee, J. Mirecki Millunchick, R. D. Twesten, D. M. Follstaedt, J. L. Reno, S. P. Ahrenkiel, and A. G. Norman, J. Mater. Sci.: Mater. Electron. 10, 191 (1999).

${ }^{14}$ P. M. Thibado, B. R. Bennett, M. E. Twigg, B. V. Shanabrook, and L. J. Whitman, J. Vac. Sci. Technol. A 14, 885 (1996).

${ }^{15}$ H. Bracht, S. P. Nicols, W. Walukiewicz, J. P. Silveira, F. Briones, and E. E. Haller, Nature (London) 408, 69 (2000).

${ }^{16}$ M. J. Cherng, H. R. Jen, C. A. Larsen, G. B. Stringfellow, H. Lundt, and P. C. Taylor, J. Cryst. Growth 77, 408 (1986).

${ }^{17}$ P. F. Fewster, Crit. Rev. Solid State Mater. Sci. 22, 69 (1997).

${ }^{18}$ E. Selvig, B. O. Fimland, T. Skauli, and R. Haakenaasen, J. Cryst. Growth 227-228, 562 (2001).

${ }^{19}$ B. R. Bennett, B. V. Shanabrook, and M. E. Twigg, J. Appl. Phys. 85, 2157 (1999).

${ }^{20}$ C. Dorin, J. Mirecki Millunchick, Y. Chen, B. G. Orr, and C. A. Pearson, Appl. Phys. Lett. 79, 4118 (2001).

${ }^{21}$ Y. H. Xie, G. H. Gilmer, C. Roland, P. J. Silverman, S. K. Buratto, J. Y. Cheng, E. A. Fitzgerald, A. R. Kortan, S. Schuppler, M. A. Marcus, and P. H. Citrin, Phys. Rev. Lett. 73, 3006 (1994).

${ }^{22}$ Z.-F. Huang and R. C. Desai (unpublished). 